\title{
Intravenous sedation for ocular surgery under local anaesthesia
}

\author{
J F Salmon, B Mets, M F M James, A D N Murray
}

\begin{abstract}
Anterior segment ophthalmic surgery is commonly performed under local anaesthesia. In order to improve patient comfort, a variety of sedation techniques has been employed in the past. The object of this study was, firstly, to determine whether continuous intravenous sedation during surgery offered any advantages in patients premedicated with temazepam and metoclopramide, and, secondly, to compare midazolam to propofol for this purpose. Forty nine patients were randomly allocated to receive no intravenous sedation $(n=15)$, continuous propofol infusion $(n=17)$, or continuous intravenous midazolam infusion $(\mathbf{n}=17)$ after peribulbar anaesthesia. Each technique provided cardiovascular and respiratory stability and allowed early recovery with minimal postoperative sequelae. Unexpected ocular field movement occurred more commonly in the patients receiving intravenous sedation, although statistical significance was not shown $(p=0.06)$. Significantly more patients in the intravenous sedation groups reported amnesia $(p=0.03)$. Patient acceptability was good irrespective of the technique used. This study suggests that continuous sedation using propofol or midazolam is not beneficial and should be avoided in ophthalmic patients who have received a simple premedication.
\end{abstract}

(Br f Ophthalmol 1992; 76: 598-601)

Retrobulbar or peribulbar anaesthesia is widely used for anterior segment ocular surgery. Local anaesthesia is cost effective, allows a rapid recovery with early hospital discharge and is popular with patients. ${ }^{12}$

It has been suggested that patient comfort might be improved during surgery by using intravenous sedation. ${ }^{3}$ Several techniques have been advocated in the past with varying success and a range of complications. ${ }^{3-6}$ The ideal sedative amnesic technique should be reliable, safe, and allow early recovery with minimal postoperative sequelae.

New drugs have become available which are ideally suited to outpatient surgery. Propofol is an intravenous anaesthetic agent which has favourable pharmacokinetic properties and is in widespread use. ${ }^{78}$ Midazolam, a new imidazobenzodiazepine, has distinct advantages over diazepam and has also been used for sedation during diagnostic and therapeutic procedures. ${ }^{9}$

Despite the excellent record of both drugs when used for intravenous sedation during other surgical procedures, to our knowledge only two trials have reported the use of midazolam and none the use of propofol, during ophthalmic surgery under local anaesthesia.$^{6}{ }^{10}$ The object of this study was to compare three groups of patients, randomly divided to receive no intravenous sedation, a continuous intravenous infusion of propofol, or a continuous intravenous infusion of midazolam during surgery. All patients received a standard oral premedication 2 hours prior to commencement of surgery.

\section{Patients and methods}

Forty nine consenting patients over the age of 50 years requiring anterior segment surgery were studied. Patients with a history of alcohol or drug abuse, an allergy to the drugs to be used in the trial, or those receiving narcotic, barbiturate, or psychotropic drugs were excluded; as were patients with epilepsy, signs of congestive cardiac failure, or evidence of renal or hepatic disease. Controlled hypertensive or diabetic patients were included.

A standard premedication of $10 \mathrm{mg}$ temazepam and $10 \mathrm{mg}$ metoclopramide was administered by mouth 2 hours prior to the commencement of surgery in all cases. The patients were divided into three groups, using a table of random numbers. One group received no intraoperative sedation, one group received a continuous intravenous infusion of propofol, and one group received a continuous intravenous infusion of midazolam during the surgery.

A peribulbar block was performed prior to the commencement of intravenous sedation by the same surgeon in a standard fashion, using a mixture of $5 \mathrm{ml}$ bupivacaine $0.5 \%, 5 \mathrm{ml}$ lignocaine $2 \%$, and hyaluronidase $1500 \mathrm{IU}$. The adequacy of the block was verified and additional local anaesthetic given if the block was incomplete. The surgeon was unaware of the method of sedation to be used.

All patient monitoring was conducted by the same anaesthetist (BM). The heart rate and rhythm were continuously monitored using a Hellige Servomed SMK ECG apparatus. A noninvasive blood pressure monitor (Dinamap, Critikon) was used to measure the blood pressure every 5 minutes. Oxygen saturation was monitored continuously (Oxyshuttle, Critikon), as was end-tidal carbon dioxide using a nasal cannula (Datex, Multicap). A stethoscope was strapped to the trachea in order to determine the development of airway obstruction with increasing sedation.

Airway obstruction was arbitrarily graded as follows: grade 1 - completely clear airway; grade 2 - slightly increased breath sounds; grade 3 noisy breathing; and grade 4 - complete obstruction requiring manual adjustment of the head position to ensure an improved airway. The level of sedation, assessed during the surgery, was 
based on the patients' response to questioning and was also arbitrarily graded into four levels: level 1 implied a patient who did not appear drowsy; level 2 a patient who was drowsy but cooperative; level 3 a patient with cooperative dysarthria; and level 4 a patient who demonstrated no vocal response to questioning.

Patients receiving no sedation during the surgery had an intravenous line inserted and received an initial bolus of normal saline, followed by a continuous saline infusion. In those patients in whom propofol was used, a slow initial bolus of propofol $1 \%$ was given to achieve cooperative dysarthria (sedation level 3 ) and then an infusion of propofol commenced at $1.0 \mathrm{mg} / \mathrm{kg} /$ hour. Patients in the third group received a slow bolus of midazolam $0.025 \%$ to achieve the same sedation level as in the propofol group and thereafter an infusion of midazolam was commenced at a rate of $1 \mathrm{mg} /$ hour. In both sedation groups, the rate of infusion was adjusted to maintain a constant level of sedation.

During the operation the anaesthetist and the surgeon independently assessed patient and operative field movement.

The infusions were stopped immediately after the completion of surgery and 5 minutes later the patient was asked prerehearsed questions (date of birth, address). The orientation of the patient was reassessed 1 hour later. The next morning, while the surgeon was still unaware of the drugs used, the patients were asked whether they had been aware of the operation and specifically whether they could remember the questions asked during the procedure. They were questioned about the presence of postoperative ocular pain, headache, nausea, their dreams of the previous night, and their subjective perception of the technique used. The intravenous site was examined.

The normally distributed data were subjected to statistical analysis using analysis of variance with the Tukey multiple range test to identify significantly different groups. Non-parametric tests were used for all other data analysis: Kruskal Wallis for comparing scores in groups of patients, and $\chi^{2}$ analysis, or Fisher's exact tests for comparing distributions: $\mathrm{p}<0.05$ was considered statistically significant.

\section{Results}

No patient was excluded from the study once the premedication had béen administered. One patient required a second injection of local anaesthetic ( $1 \mathrm{ml}$ retrobulbar lignocaine $2 \%$ ) for

Table 1 Patient dato

\begin{tabular}{llll}
\hline & $\begin{array}{l}\text { Saline } \\
(n=15)\end{array}$ & $\begin{array}{l}\text { Midazolam } \\
(n=17)\end{array}$ & $\begin{array}{l}\text { Propofol } \\
(n=17)\end{array}$ \\
\hline $\begin{array}{l}\text { Sex } \\
\text { Male }\end{array}$ & 9 & 8 & 7 \\
$\quad$ Female & 6 & 9 & 10 \\
$\begin{array}{l}\text { Race } \\
\text { White }\end{array}$ & 3 & 5 & 2 \\
$\quad \begin{array}{l}\text { Mixed } \\
\text { Black }\end{array}$ & 8 & 10 & 13 \\
$\begin{array}{l}\text { Age (mean in years) } \\
\text { Weight (kg) }\end{array}$ & 4 & 2 & 2 \\
$\begin{array}{l}\text { Duration of operation } \\
\text { (minutes) (SD) }\end{array}$ & $70 \cdot 0$ & $64 \cdot 5$ & $66 \cdot 5$ \\
& $38 \cdot 0(2 \cdot 2)$ & $39 \cdot 6(2 \cdot 1)$ & $39 \cdot 7(1 \cdot 9)$ \\
\hline
\end{tabular}

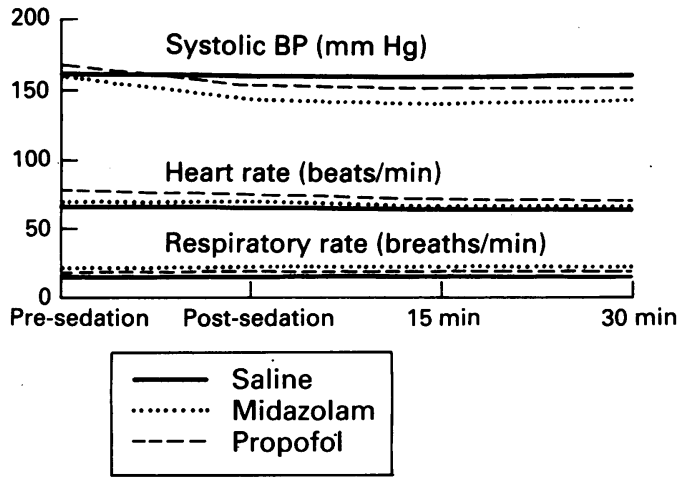

Figure 1 Cardiovascular and respiratory changes during the procedure in the three groups. There are no significant differences.

an incomplete block, one had a small abduction movement, and one had pain with the insertion of a superior rectus bridal suture. All the others had good akinesia and local analgesia.

The three groups were statistically comparable and the mean duration of the procedures was similar in each group (Table 1). The mean total dose of midazolam was $1.41 \mathrm{mg}$ (standard error 0.13 ) and the mean infusion rate was 1.04 $\mathrm{mg}$ /hour (standard error 0.03 ). The mean total dose of propofol was $43.2 \mathrm{mg}$ (standard error 4.0) and the infusion rate was $7.7 \mathrm{ml}$ per hour (standard error $0 \cdot 4$ ).

There were no significant differences between the three groups in respect of cardiovascular variables (Fig 1). Though $47 \%$ of patients (8/17) receiving intravenous propofol had a rise or a drop of systolic blood pressure of more than 20 $\mathrm{mm} \mathrm{Hg}$, compared with $29 \cdot 4 \%$ (5/17) receiving midazolam and $20 \%(3 / 15)$ receiving saline infusion, this was not statistically significant. A pulse rate drop of more than 20 beats/minute was noted in two patients receiving intravenous propofol.

Minor airway obstruction (snoring) occurred with equal frequency in the midazolam group and the propofol group, but this was not statistically significant when compared with the saline group (Fig 2). One patient receiving propofol

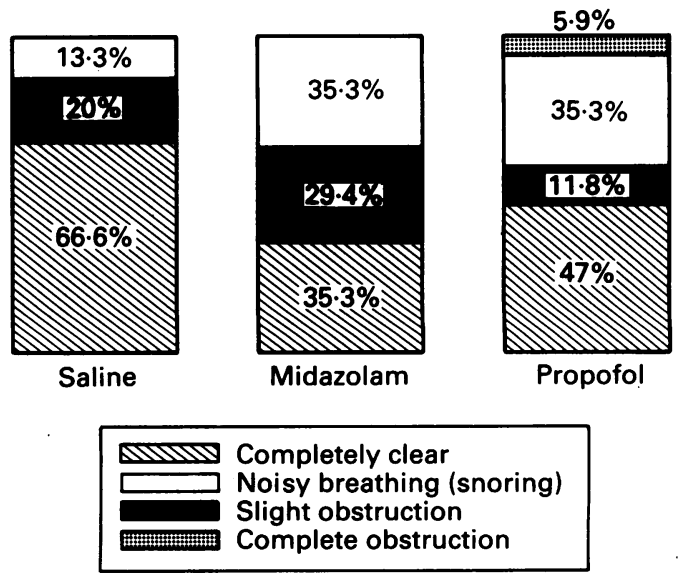

Figure 2 The incidence and severity of airway obstruction in each group. There are no significant differences. Grading: 1completely clear; 2 - slightly increased breath sounds; 3 - noisy breathing (snoring); 4-complete obstruction requiring manual airway correction. 


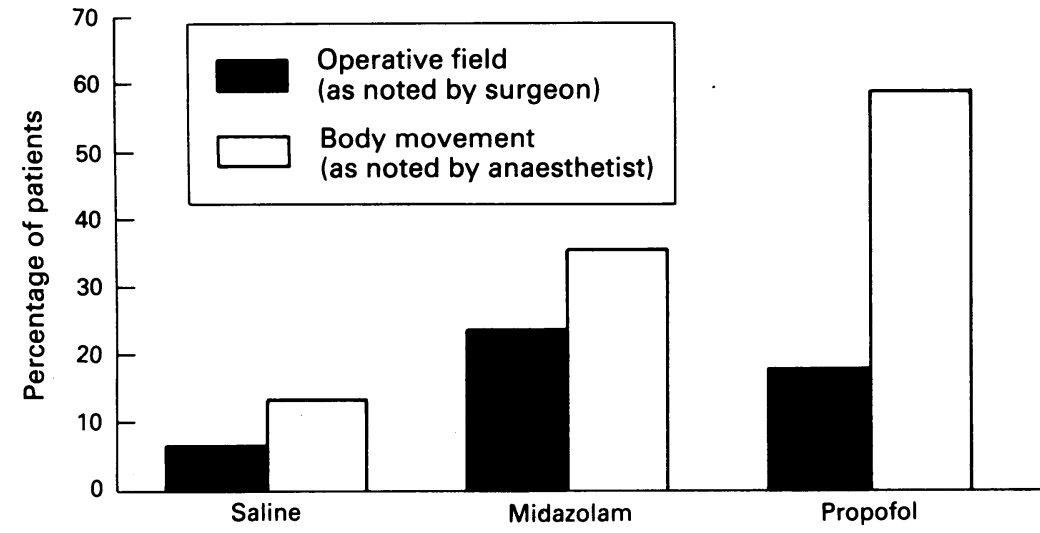

Figure 3 Incidence of movement noted by surgeon or anaesthetist. Incidence of patient movement noted by anaesthetist (fidgetting) was significantly greater in the propofol group than in the saline group.
Table 2 Patients'opinions of the sedation technique

\begin{tabular}{lccc}
\hline & $\begin{array}{l}\text { Saline } \\
(n=15)\end{array}$ & $\begin{array}{l}\text { Midazolam } \\
(n=17)\end{array}$ & $\begin{array}{l}\text { Propofol } \\
(n=17)\end{array}$ \\
\hline Operative amnesia & $0(0 \%)$ & $5(29 \cdot 4 \%)$ & $6(35 \cdot 3 \%)$ \\
Postop eye pain & $1(6 \cdot 6 \%)$ & $0(0 \%)$ & $1(5 \cdot 95 \%)$ \\
Postop headache & $4(26 \cdot 6 \%)$ & $6(35 \cdot 3 \%)$ & $8(47 \cdot 1 \%)$ \\
Postop nausea & $0(0 \%)$ & $0(0 \%)$ & $2(11 \cdot 8 \%)$ \\
Postop dreams & $4(26 \cdot 65 \%)$ & $2(11 \cdot 8 \%)$ & $2(11 \cdot 8 \%)$ \\
Same technique again & $14(93 \cdot 3 \%)$ & $13(76 \cdot 5 \%)$ & $17(100 \%)$ \\
Satisfaction & $15(100 \%)$ & $15(88 \cdot 2 \%)$ & $17(100 \%)$ \\
\hline
\end{tabular}

similar technique for another procedure, than those in the propofol group (17/17). This was statistically significant $(p=0.031)$. When comparing those patients who had received no intravenous sedation with those who had received intravenous sedation, patients were equally satisfied irrespective of the technique used (Table 2).

had a complete airway obstruction which necessitated repositioning of the neck. There was no significant difference between the three groups in terms of the minimum oxygen saturation or the maximum end tidal $p \mathrm{CO}_{2}$ and in all patients these parameters remained satisfactory throughout the surgery. In one patient in each group the minimum oxygen saturation dropped below $95 \%$. In one patient receiving midazolam the minimum saturation was $89 \%$, in one receiving propofol $91 \%$, and in one receiving saline $93 \%$. In two of these patients this was clearly related to minor airway obstruction.

The anaesthetist noted more unexpected patient movement in the propofol group and in the midazolam group than in the saline group (Fig 3). There was a significantly greater incidence of movement in the group receiving propofol compared with the saline group $(\mathrm{p}=$ $0 \cdot 009$ ). Movement of the operative field was noted by the surgeon in one patient in the saline group who had a small abduction movement related to an inadequate block. One of four patients in the midazolam group and three patients in the propofol group had a major unexpected head movement, but in no patient was the surgery compromised (Fig 3). Statistical significance was not demonstrated when operative field movement was compared in patients receiving intravenous sedation with those not receiving intravenous sedation $(\mathrm{p}=0.06)$.

Five minutes after stopping the infusion each patient in all three groups was able to answer prerehearsed questions and was aware of his surroundings. All patients remained fully orientated when assessed in the recovery room 1 hour later.

There was no significant difference between the three groups in terms of postoperative pain, nausea, headaches, or dreams when assessed the following morning (Table 2). No patient recalled experiencing pain with the intravenous infusion of the previous day, but one patient who had received intravenous midazolam was found to have localized tenderness over the intravenous site. Patients receiving propofol or midazolam were less aware of the operation than patients receiving saline $(\mathrm{p}=0.03$ and $\mathrm{p}=0.02$ respectively) (Table 2). Fewer patients in the midazolam group (13/17) would have chosen a

\section{Discussion}

Many single and multiple drug sedation regimens have been advocated for use during ophthalmic surgery under local anaesthesia. Intravenous propofol and midazolam have been successfully used for sedation during other surgical and diagnostic procedures under regional anaesthesia. ${ }^{11-15}$ It has been our practice to use a simple oral premedication of temazepam and metoclopramide 2 hours prior to surgery. The object of this study was to examine whether there would be any advantage in using additional intravenous sedation using the new drugs, propofol, and midazolam.

The greatest potential advantage of propofol is early recovery with rapid return to normal psychomotor function. ${ }^{78}$ There is a low incidence of postoperative nausea and the drug has reliable amnestic properties. For the ophthalmologist, a potential benefit during anterior segment surgery is a significant decrease in the intraocular pressure. ${ }^{16}$ When used as a continuous sedation technique, the infusion rates need to be determined for each patient and have to be adjusted during surgery to ensure that sedation does not become too light or does not progress into full anaesthesia. ${ }^{17}$

Midazolam has the anxiolytic, hypnotic, anticonvulsant, muscle relaxant, and anterograde amnestic effects characteristic of benzodiazepines. ${ }^{9}$ The drug is effective in reducing the intraocular pressure. ${ }^{10}$ Compared with diazepam, midazolam produces less postoperative drowsiness, more amnesia, and less venous irritation. Recovery from midazolam appears to be less rapid than recovery from propofol. ${ }^{15}$

As expected from the pharmacokinetic characteristics of both drugs, there was rapid reversibility of action and all patients in both intravenous sedation groups were aware of their surroundings and could correctly answer questions 5 minutes after the drugs were stopped. It was statistically significant that more patients who had received midazolam and propofol were unaware of the operation than those receiving a premedication alone.

There were no significant differences between the three groups in terms of cardiovascular stability, oxygen saturation, and expiratory 
levels of $p \mathrm{CO}_{2}$. While $35.3 \%$ of patients in the midazolam group and $41.2 \%$ of patients in the propofol group had a limited degree of airway obstruction, this was not significant when compared to patients receiving no intravenous sedation. One patient receiving propofol had a complete obstruction of the airway which necessitated repositioning of the neck. These findings differ from a previous trial comparing propofol with midazolam by infusion, where 'excellent' airway maintenance was found..$^{15}$

During ophthalmic surgery under local anaesthesia an uncontrolled and unexpected movement of the head, and consequently movement of the operative field, could result in major surgical complications. The anaesthetist noted more body movement in the propofol group and in the midazolam group than in the saline group. However, statistical significance was only demonstrated for the propofol group. In both intravenous sedation groups the surgeon noted head movement, including a major movement in one patient receiving midazolam and in three patients receiving propofol. This occurred because the patients fell asleep and awoke unaware of their surroundings or because communication was reduced. Studies comparing intravenous propofol and midazolam have reported similar problems. ${ }^{14}$ is Despite the excellent record of these drugs in other forms of surgery performed under local anaesthesia, this single side effect makes the technique of sedation as described in this study undesirable for ophthalmic surgery.

On assessment of the patients' wish to have a second procedure using a similar technique, there was no difference statistically between those who had received intravenous sedation and those who had received no intravenous sedation. Except for two patients who had received midazolam, patients were satisfied irrespective of the technique used.

This study suggests that continuous intravenous sedation with propofol or midazolam is not beneficial in well prepared and informed patients who have received a simple premedication of temazepam and metoclopramide. In our opinion, the ideal patient undergoing ophthalmic surgery is conscious, cooperative, and pain free. The techniques described in this report do not consistently produce these conditions and should therefore be avoided in ophthalmic patients.

1 Ogg TW, Obey PA. The workload of a purpose built day surgical unit. Ann R Coll Surg 1987; 69: 110-2.

Wats MT, Pearce JL. Day-case cataract surgery. Brf Ophthalmol 1988; 72:897-9.

3 Ingram HV, Davison MHA. Intraocular surgery with local analgesia and heavy sedation. Lancet 1961; i: $1321-4$.

4 Wine NA. Sedation with neuroleptanalgesia in cataract surgery. Am $\mathcal{F}$ Ophthalmol 1966; 61: 456-60.
.

5 Gilbert J, Holt JE, Johnston J, Sabo BA, Weaver JS Intravenous sedation for cataract surgery. Anaesthesia 1987

6 Chung F, Cheng DCH, Seyone C, Dyck BJ. A randomized comparison of midazolam and diazepam injectable emulsion in cataract surgery. Can $\mathcal{F}$ Anaesth 1990; 37: 528-33.

7 Langley MS, Heel RC. Propofol: a review of its pharmacodynamic and pharmacokinetic properties and use as an intravenous anesthetic. Drugs 1988; 35: 334-72.

8 Sebel PS, Lowdon JD. Propofol: a new intravenou anesthetic. Anesthesiology 1989; 71: 260-77.

9 Reves JG, Fragen RJ, Vinik R, Greenblatt DJ. Midazolam pharmacology and uses. Anesthesiology 1985; 62: 310-24.

10 Gobeaux D, Sardnal F. Midazolam and flumazenil in ophtha mology. Acta Anaesthesiol Scand (Suppl) 1990; 34: 35-8.

11 Mackenzie N, Grant IS. Propofol for intravenous sedation. Anaesthesia 1987; 42: 3-

12 McClure JH, Brown DT, Wildsmith JAW. Comparison of the iv administration of midazolam and diazepam as sedation

13 Magni VC, Frost RA, Leung JWC, Cotton PB. A randomized comparison of midazolam and diazepam for sedation in upper gastrointestinal endoscopy. Anaesthesia 1983; 55: upper gas

14 Fanard $L$, Van Steenberge A, Demeire $X$, van der Puyl F. Comparison between propofol and midazolam as sedative agents for surgery under regional anaesthesia. Anaesthesia agents for surgery under

15 Wilson E, Mackenzie N, Grant IS. A comparison of propofol and midazolam by infusion to provide sedation in patients who receive spinal anaesthesia. Anaesthesia $1988 ; 43$ (suppl): $91-4$

16 Guedes Y, Rakotoseheno JC, Leveque M, Mimouni F, Egreteau JP. Changes in intraocular pressure in the elderly during anaesthesia with propofol. Anaesthesia 1988; 43 (suppl): 58-60.

17 Gepts E, Claeys MA, Camu G, Smekens L. Infusion of propofol ('Diprivan') as a sedative technique for colonoscopies. Postgrad Med f 1985; 61 (suppl): 120-6. 